\title{
Combination of PI3K and MEK inhibitors yields durable remission in PDX models of PIK3CA-mutated metaplastic breast cancers
}

F. Coussy ${ }^{1,2,3^{*}} \mathbb{D}$, R. El Botty ${ }^{2}$, M. Lavigne ${ }^{4}$, C. Gu${ }^{4}$, L. Fuhrmann ${ }^{4}$, A. Briaux ${ }^{1}$, L. de Koning ${ }^{5}$, A. Dahmani ${ }^{2}$, E. Montaudon ${ }^{2}$, L. Morisset ${ }^{2}$, L. Huguet ${ }^{2}$, L. Sourd ${ }^{2}$, P. Painsec ${ }^{2}$, S. Chateau-Joubert ${ }^{6}$, T. Larcher ${ }^{7}$, S. Vacher ${ }^{1}$, S. Melaabi ${ }^{1}$, A. Vincent Salomon ${ }^{4}$, E. Marangoni ${ }^{2+}$ and I. Bieche ${ }^{1,8+}$

\begin{abstract}
Background: Metaplastic breast cancer (MBC) is a rare form of breast cancer characterized by an aggressive clinical presentation, with a poor response to standard chemotherapy. MBCs are typically triple-negative breast cancers (TNBCs), frequently with alterations to genes of the PI3K-AKT-mTOR and RTK-MAPK signaling pathways. The objective of this study was to determine the response to PI3K and MAPK pathway inhibitors in patient-derived xenografts (PDXs) of MBCs with targetable alterations.

Methods: We compared survival between triple-negative MBCs and other histological subtypes, in a clinical cohort of 323 TNBC patients. PDX models were established from primary breast tumors classified as MBC. PI3K-AKT-mTOR and RTK-MAPK pathway alterations were detected by targeted next-generation sequencing (NGS) and analyses of copy number alterations. Activation of the PI3K-AKT-mTOR and RTK-MAPK signaling pathways was analyzed with reverse-phase protein arrays (RPPA). PDXs carrying an activating mutation of PIK3CA and genomic changes to the RTK-MAPK signaling pathways were treated with a combination consisting of a PI3K inhibitor and a MEK inhibitor.

Results: In our clinical cohort, the patients with MBC had a worse prognosis than those with other histological subtypes. We established nine metaplastic TNBC PDXs. Three had a pathogenic mutation of PIK3CA and additional alterations to genes associated with RTK-MAPK signaling. The MBC PDXs expressed typical EMT and stem cell genes and were of the mesenchymal or mesenchymal stem-like TNBC subtypes. On histological analysis, MBC PDXs presented squamous or chondroid differentiation. RPPA analysis showed activation of the PI3K-AKT-mTOR and RTKMAPK signaling pathways. In vivo, the combination of PI3K and MAPK inhibitors displayed marked antitumor activity in PDXs carrying genomic alterations of PIK3CA, AKT1, BRAF, and FGFR4.

Conclusion: The treatment of metaplastic breast cancer PDXs by activation of the PI3K-AKT-mTOR and RTK-MAPK pathways at the genomic and protein levels with a combination of PI3K and MEK inhibitors resulted in tumor regression in mutated models and may therefore be of interest for therapeutic purposes.
\end{abstract}

Keywords: Metaplastic breast cancer, PI3K inhibitor, MEK inhibitor, Combination of targeted therapies

\footnotetext{
* Correspondence: florence.coussy@curie.fr

${ }^{\dagger} \mathrm{E}$. Marangoni and I. Bieche contributed equally to this work.

'Unit of Pharmacogenomics, Department of Genetics, Institut Curie, Paris,

France

${ }^{2}$ Laboratory of Preclinical Investigation, Department of Translational Research,

Institut Curie Research Center, Paris, France

Full list of author information is available at the end of the article
}

(c) The Author(s). 2020 Open Access This article is distributed under the terms of the Creative Commons Attribution 4.0 International License (http://creativecommons.org/licenses/by/4.0/), which permits unrestricted use, distribution, and reproduction in any medium, provided you give appropriate credit to the original author(s) and the source, provide a link to the Creative Commons license, and indicate if changes were made. The Creative Commons Public Domain Dedication waiver (http://creativecommons.org/publicdomain/zero/1.0/) applies to the data made available in this article, unless otherwise stated. 


\section{Introduction}

Metaplastic breast carcinoma $(\mathrm{MBC})$ is a rare, heterogeneous group of breast cancers characterized by differentiation of the neoplastic epithelium into squamous and/or mesenchymal elements, including spindle, chondroid, osseous, and rhabdomyoid cells. These tumors may consist entirely of metaplastic elements or be composed of a complex mixture of carcinoma and metaplastic areas $[1,2]$. Most MBCs have a triple-negative phenotype, with no estrogen receptor (ER) or progesterone receptor (PR) expression and no overexpression of ERBB2 [2, 3]. The clinical presentation of $\mathrm{MBC}$ is characterized by a rapidly growing tumor mass at diagnosis, with a higher incidence of stage III and IV disease and a higher risk of local recurrence than for invasive ductal carcinomas $[4,5]$. $\mathrm{MBC}$ is typically chemoresistant. Neoadjuvant chemotherapy and metastatic treatment are of limited efficacy for reducing tumor burden and preventing disease progression [6-8]. Survival is lower in MBC patients than in non-MBC patients $[7,9]$.

Gene expression analyses have shown that most MBCs are of the mesenchymal-like and mesenchymal stem-like molecular subtypes, according to the classification of triple-negative breast cancers [10].

The rarity of $\mathrm{MBC}$ has limited opportunities for characterizing the molecular genetic landscape in large cohorts of tumors, but several small case series have been analyzed, and molecular details are beginning to emerge [11]. Several studies have demonstrated a high frequency of phosphoinositide (PI)-3 kinase pathway aberrations, including frequent PIK3CA mutations and TP53 mutations [12]. Other changes, such as CDKN2A loss and EGFR amplification, have been described, but at low frequency [12-14]. Krings et al. sequenced a panel of 28 MBCs and found strong enrichment in aberrations of the PIK3CA/PIK3R1 (61\%) and RAS-MAP kinase (25\%) pathways, affecting $H R A S, K R A S$, and NF1 in particular [15]. Similarly, McCart et al. performed whole-exome sequencing on 30 cases and found mutations of TP53, PTEN, and PIK3CA and an overrepresentation of NF1 mutations [1].

Alterations to the PI3K-AKT-mTOR pathways are potentially promising targets for $\mathrm{MBC}$ management. However, no clinical data for PI3K inhibitor treatment have been reported for $\mathrm{MBC}$ patients, due to the rarity of these tumors, and preclinical data for $\mathrm{MBC}$ patient-derived xenografts are also lacking.

We report here the establishment and molecular characterization of MBC PDXs. We show, for the first time, that a combination of PI3K and MEK inhibitors is highly effective against MBC PDXs with PIK3CA mutations and alterations to the RTK-MAPK signaling pathway.

\section{Materials and methods}

\section{Clinical cohort}

Samples from 323 unilateral invasive triple-negative primary breast tumors excised from women managed at Institut Curie (Paris and Saint-Cloud, France) between 1980 and 2015 were analyzed (Additional file 1: Table S1). Most patients (67\%) were diagnosed and treated after 2000. All patients admitted to our institution before 2007 were informed that their tumor samples might be used for scientific purposes and were given the opportunity to refuse such use. Since 2007, patients admitted to our institution also give express consent for the use of their samples for research purposes, by signing an informed consent form. Patients (mean age, 56 years; range, 28-91 years) met the following criteria: primary unilateral non-metastatic TNBC, with full clinical, histological, and biological data and full follow-up at Institut Curie. Median follow-up was 7.8 years (range 8 months to 36 years). Seventy-eight patients had developed metastases within 10 years.

\section{Patient-derived xenografts}

PDXs were established from the engraftment of primary breast tumors with a procedure described elsewhere [16-18]. Female Swiss nude mice were purchased from Charles River Laboratories and maintained under specific pathogen-free conditions. The experimental protocol and animal housing were in accordance with institutional guidelines and with the recommendations of the French Ethics Committee (Agreement B75-05-18, France). Three metaplastic TNBC PDXs with genomic alterations were chosen for experimental analysis: HBCx-60, HBCx-165, and HBCx-178. BYL-719 (PI3K inhibitor) and selumetinib (MEK inhibitor) were purchased from Medchem Express. BYL-719 was administered five times per week, at doses of $35 \mathrm{mg} / \mathrm{kg}$, by oral gavage. Selumetinib (MEK inhibitor) was administered five times per week, at doses of $100 \mathrm{mg} / \mathrm{kg}$ (50 mg/kg, bid), by oral gavage. Adriamycin (DOX, doxorubicin, Teva Pharmaceuticals) and cyclophosphamide (Endoxan, Baxter) were administered by the intraperitoneal (i.p.) route, at doses of 2 and $100 \mathrm{mg} / \mathrm{kg}$, respectively, every 3 weeks. We included 10 mice per groups. Tumor growth was evaluated by measuring two perpendicular tumor diameters with calipers twice weekly. Individual tumor volumes were calculated as follows: $V=(a \times b)^{2} / 2$, where " $a$ " is the largest diameter, and " $b$ " is the smallest diameter. For each tumor, volumes are expressed relative to the initial volume, as a relative tumor volume (RTV). Tumor growth inhibition (TGI) was assessed by dividing mean RTV (relative tumor volume) in the treated group by mean RTV in the control group at the same time. The statistical significance of TGI was assessed by comparing tumor volumes between the treated and control groups in paired Student's $t$ tests. Stable disease was defined as the percentage change in volume, between 0 and -50 . 


\section{Transcriptomic data analysis}

We used gene expression arrays for the transcriptomic profiling of 64 PDX TNBCs. The concentration and integrity/purity of each RNA sample were assessed with the RNA 6000 LabChip kit (Agilent) and an Agilent 2100 bioanalyzer. GeneChip Human 1.1 ST arrays were hybridized according to Affymetrix recommendations, with the WT Expression Kit protocol (Life Technologies) and Affymetrix labeling and hybridization kits. Arrays were normalized according to the RMA normalization procedure, with the oligo package [19]. No additional human-mouse cross-hybridization filtering was applied, as our xenograft samples contained less than $5 \%$ mouse cells (percentage determined by RT-PCR quantifying transcripts of the ubiquitously expressed TBP gene with specific mouse and human primers pairs), a percentage too low to affect the expression profiles obtained with HuGene1.0 arrays [20]. The TNBC molecular subtypes of the PDXs were determined from gene expression data, with the TNBCtype software developed by Chen et al [21].

\section{Somatic mutation analysis}

We analyzed 64 PDXs by the targeted NGS of 95 genes, chosen from the genes most frequently mutated in breast cancer $(>1 \%)$ and including potential therapeutic targets, as previously described [22]. Briefly, NGS was performed on an Illumina HiSeq 2500 sequencer, and the genomic variants were annotated with the COSMIC and 1000 genome databases. Variants with a low allelic frequency $(<5 \%)$ or low coverage $(<100 x)$ were excluded from the analysis. Deleterious genomic alterations were defined as follows: (i) for oncogenes, only gain-of-function mutations were considered (i.e., hotspot missense mutations, in-frame insertions/deletions/splicing reported to be oncogenic), and (ii) for tumor suppressor genes (TSG), only loss-of-function mutations were considered (i.e., biallelic truncating alterations (nonsense mutations, frameshift insertions/deletions/splicing) or monoallelic truncating alterations associated with heterozygous deletion detected by copy number analysis). Genomic variants were biologically validated by comparison with the COSMIC, TumorPortal, and cBioportal databases $[7,15]$.

\section{Somatic copy number alteration (SCNA) analysis}

PDXs were profiled with Affymetrix genomics arrays: 24 with SNP 6.0 and 37 with the Cytoscan HD array. Genome-wide copy number analysis was performed with Affymetrix SNP arrays, as previously described [23, 24]. SNP 6.0 or Cytoscan HD arrays were used with $500 \mathrm{ng}$ and $250 \mathrm{ng}$ of gDNA, respectively, as the input material, as recommended by the manufacturer. Raw data were normalized with Genotyping console (SNP6.0 arrays) or Chromosome Analysis Suite (Cytoscan HD arrays). The focal amplification of oncogenes was defined as a $\log$ ratio $>1.58$ ( 6 copies per diploid genome) and a maximum size of $<10 \mathrm{Mb}$. The biallelic inactivation of TSGs was defined as homozygous deletion or truncating mutations associated with heterozygous deletion. Copy number alterations were compared with cBioPortal data for TCGA breast cancer $[25,26]$. All PDX copy numbers are represented by the Circular Binary Segmentation algorithm [27], as implemented in the DNAcopy package for R, with a minimum width of 3 , an alpha risk of $1 \%$, and up to 10 , 000 permutations. Downstream analysis of the sample population was performed with GISTIC2.0 [28], with default settings. CGH explorer and CGHcall were used for visual representation of the results and figures [29]. The BRCAness signature consisted of large-scale state transitions (LST), defined as chromosomal breaks between adjacent regions of at least $10 \mathrm{Mb}$ initially described by Popova et al. with Gap methodology [30].

\section{RT-qPCR in PDXs}

Total RNA extraction and RT-qPCR have been described elsewhere [31]. The TBP gene (GenBank accession no. NM_003194) encoding the TATA box-binding protein (a component of the DNA-binding protein complex TFIID) was quantified as an endogenous RNA control, and each sample was normalized on the basis of its TBP content [28]. $\mathrm{N}$-fold differences in target gene expression relative to the TBP gene ("Ntarget"), were determined as Ntarget $=2^{\Delta \text { Ctsample }}$, where the $\Delta \mathrm{Ct}$ value of the sample was obtained by subtracting the mean $\mathrm{Ct}$ value of the target gene from that of the TBP gene [32].

For the gene expression study in PDXs, mRNA levels were normalized to obtain a "basal mRNA level" (smallest amount of mRNA quantifiable $(\mathrm{Ct}=35)$ ) equal to 1 . We analyzed the expression of genes involved in epithelial-mesenchymal transition (EMT) (SNAI2, VIM, ACTA2, SPARC, TCF7L2, CAV1) in all TNBC PDXs and included 49 with well-known transcriptomic classifications in the final analysis.

\section{Reverse-phase protein array (RPPA)}

RPPA was performed as previously described [33] for 48 of the 64 TNBC PDXs (16 recent PDXs were not included in the RPPA analysis). We assessed pathway activation, by calculating a PI3K-AKT-mTOR and RTK-MAPK pathway score with normalized data. This score was obtained by calculating the sum of the protein levels for positive components and subtracting that for the negative components of the pathway (especially for PI3K-AKT-mTOR pathway): (i) PI3K p110 subunit $\beta$, P-AKT1 (Ser473), P-AKT1 (Thr308), P-4E-BP1, P-p70 S6 kinase, P-S6 ribosomal protein minus PTEN (Cell Signaling Technology ${ }^{\circ}$ ), and (ii) PRSK2 (Cell Signaling Technology ${ }^{\circ}$ ). 


\section{Immunohistochemistry (IHC)}

Tumors from patients and xenografts were fixed in 10\% neutral-buffered formalin, embedded in paraffin, and stained with hematoxylin and eosin. The same histologist (ML) compared morphology between patient and PDX tumors. Four representative MBCs with chondroid and squamous characteristics were compared ( $\mathrm{HBCx}-$ 130, HBCx-162, HBCx-165, HBCx-178).

\section{Western blot analysis}

Proteins were extracted from tumors using a Laemmli buffer (50 mM Tris HCL pH 8, 2 mM DTT, 2\% SDS, 5\% glycerol), supplemented with protease and phosphatase inhibitors. Lysates were resolved on 10\% agarose gels, transferred onto nitrocellulose membranes (Bio-Rad, Hercules, CA, USA), and immunoblotted with rabbit antibodies against AKT, p-AKT (ser 473), S6, p-S6 (Ser235/ 236), ERK, p-ERK (Thr202/Tyr204), MEK, p-MEK (Ser217/221), and GAPDH (Cell Signaling $\left.{ }^{\circ}\right)$. After washes, membranes were incubated with the appropriate horseradish peroxidase-conjugated affinity-purified goat antirabbit secondary antibodies (Jackson ImmunoResearch Laboratories, Inc., Interchim).

\section{Screening for PIK3CA mutations in patients}

Hotspot PIK3CA mutations (exons 1, 2, 9, 20) were detected by sequencing cDNA fragments obtained by RT-PCR amplification. The exons of the gene to be screened were chosen on the basis of the mutation frequency reported in COSMIC: Catalogue of Somatic Mutations in Cancer (cancer.sanger.ac.uk/). Screening was performed by high-resolution melting curve analysis on a LightCycler 480 (Roche Diagnostics, Penzberg, Germany), with LCGreen Plus + Melting Dye fluorescence (Biotech, Idaho Technology Inc., Salt Lake City, UT). Details of the primers and PCR conditions used are available on request. The amplified products were sequenced with the BigDye Terminator kit on an ABI Prism 3130 automatic DNA sequencer (Applied Biosystems, Courtaboeuf, France) with a detection sensitivity of 5\% mutated cells, and the sequences obtained were compared with the corresponding reference cDNA sequences (PIK3CA NM 006218). All mutations detected were confirmed in a second independent sequencing run.

\section{Statistical analysis}

We compared the RT-qPCR values obtained for the MSL and M subtypes with those of other subtypes, in $t$ tests. The proportions of genomic alterations between metaplastic and other types of PDX were compared in Fisher's exact tests.

Metastasis-free survival (MFS) was assessed by determining the interval between diagnosis and the detection of the first distant metastasis. Overall survival (OS) was determined as the interval between diagnosis and death. Survival was estimated by the Kaplan-Meier method.

\section{Results}

Metaplastic breast cancer patients have a worse prognosis than patients with other histological subtypes We compared survival between triple-negative $\mathrm{MBC}$ and other histological subtypes in a clinical cohort of 323 TNBC patients treated at our institute, with a long follow-up. This cohort included 13 MBCs (4\%), 43 apocrine BCs (13.3\%), 36 medullary BCs (11.1\%), 198 breast cancers of no special type (NST) (61.4\%), and other rare forms of TNBC (10.2\%) (oncocytic, acinic, lobular, adenoid cystic papillary, micropapillary, or mucinous). MBC patients had a worse prognosis than patients with other subtypes, in terms of metastasis-free survival (MFS) and overall survival (OS) ( $p=0.02$ and $p=0.01$, respectively) (Fig. 1). The medullary TNBC subgroup had a particularly good prognosis.

We investigated the frequency of tumors with PIK3CA mutations with Sanger sequencing of PIK3CA. In the whole cohort of tumors, PIK3CA mutations were present in $15 \%$ of metaplastic tumors and $12.2 \%$ of the tumors of other subtypes $(p=0.66)$ (NST, 13\%; apocrine, $14 \%$; rare, $12 \%$; medullary, $0 \%)$. PIK3CA mutations were of no prognostic values in the metaplastic subtype (data not shown).

\section{PDX models of metaplastic breast cancer are characterized by a mesenchymal phenotype}

In our cohort of 64TNBC PDX models (the description of the first 61 PDX were recently published [22]), nine displayed metaplastic differentiation (14\%) (Table 1). Three displayed fusiform differentiation, three had squamous characteristics, and three had mixed components (chondroid-fusiform or squamous-fusiform). Figure 2a shows the morphological features of four MBCs (patients and PDXs): HBCx-162, HBCx-130, HBCx-165, and HBCx178 . HBCx-162 was characterized by an abundant chondromyxoid matrix, and HBCx-130, HBCx-165, and HBCx-178 were squamous cell MBCs. The specific morphological characteristics of the initial tumors were also present in the corresponding PDXs. Squamous cell metaplastic carcinomas have a particular set of characteristics: tumor masses bordering cystic cavities, high degrees of nuclear pleomorphism, large polygonal cells with abundant eosinophilic cytoplasm, keratinization beads sometimes present, and abundant inflammatory infiltrate. These highly specific aspects are found in both PDXs and patient tumors. A similar phenomenon is observed for metaplastic carcinomas with mesenchymal chondroid differentiation: each pair of tumors displays an abundant cartilaginous and myxoid matrix enclosing the carcinomatous cells. 

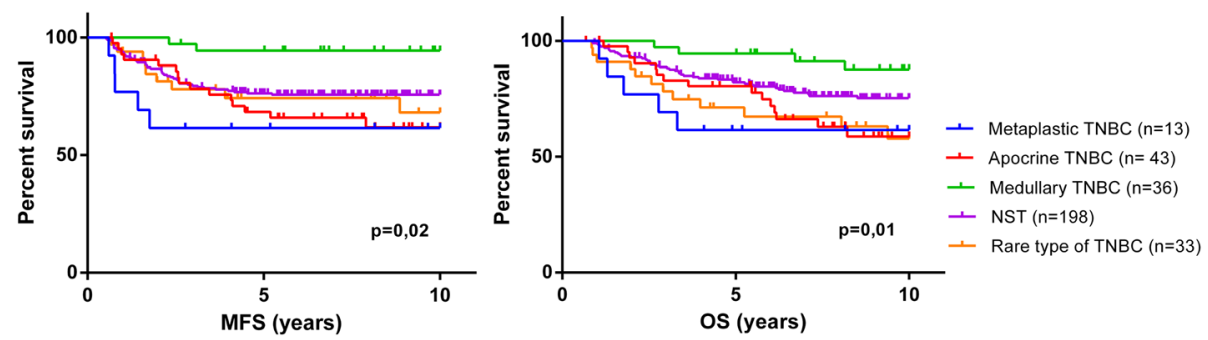

Fig. 1 Metastasis-free survival (MFS) and overall survival (OS) in 323 different subtypes of TNBC (198 of no special type, 43 apocrine, 36 medullary, 13 metaplastic, and 33 rare TNBCs) analyzed in log-rank tests

Nine MBC PDXs were characterized at the transcriptomic level: four were classified as $\mathrm{M}$ (mesenchymal) or MSL (mesenchymal stem-like), 1 as BL2 (basal-like 2), and 3 were unstable. For further characterization of the phenotype of MBC PDXs, we performed a RT-PCR analysis of various genes involved in the epithelialmesenchymal transition (EMT), a cancer cell phenotype previously associated with MBC [34], in 48 TNBC PDXs. We generated an expression heatmap for the $S N A I 2$, ACTA2, VIM, SPARC, and TCF7L2 genes in the various TNBC transcriptomic subtypes (14 unstable and 5 NA tumors were excluded from the analysis) (Fig. 2b). The expression of the SNAI2, ACTA2, VIM, SPARC, and TCF7L2 genes was significantly stronger in the MSL/M subtypes than in the other subtypes (unpaired $t$ test).

\section{Activation of the PI3K-AKT-mTOR and RTK-MAPK pathways in MBC PDX}

The genomic alterations (mutations and copy number alterations) found in MBC PDXs concerned the PI3K and RTK-MAPK pathways.

Figure 3a shows the major genomic alterations in metaplastic PDX. Eight genetic alterations affecting the PI3K-AKT-mTOR signaling pathway were identified in six of the nine metaplastic PDX, including four PIK3CA mutations (44.4\%), three PTEN genetic alterations (33.3\%), and one AKT1 amplification (11.1\%). PIK3CA (activating mutations) was significantly more frequently altered in metaplastic TNCB than in other subtypes (Fisher's test, $p=0.01$ ) (Fig. 3b). Five of the nine metaplastic PDX (55\%) harbored one alteration to the TP53 gene ( $50 \%$ for other subtypes). The additional genetic alterations of theranostic interest were two alterations to the $B R A F$ gene (one mutation and one focal amplification), two focal amplifications of FGFR4, and one focal amplification of FGFR1, EGFR, and MET. Four of the nine (44\%) characterized MBCs displayed genomic changes to both the PI3K-AKT-mTOR and RTK-MAPK pathways (versus 11/55 for other subtypes, 20\%).
We confirmed the activation of the PI3K-AKT-mTOR and RTK-MAPK signaling pathways at the protein level, by analyzing the expression of the major effectors of the PI3K-AKT-mTOR pathway (PI3-kinase, p-AKT, p-4EBP1, p70-S6-kinase, p-S6RP, PTEN) and the RTK-MAPK pathway (p-MEK1, p-RSK2) by RPPA analysis. This analysis confirmed the activation of the PI3K pathway in metaplastic BCs. RSK2 is an effector of the MAPK pathway. Most metaplastic BCs had high RSK2 scores. However, by contrast to the PI3K pathway, the results of genomic and protein analysis were not well correlated (Additional file 2: Figure S1).

\section{PIK3CA-mutated PDXs respond to the combination of PIK3CA and MEK inhibitors}

Based on the high frequency of concomitant alterations of the PI3K-AKT-mTOR and RTK-MAPK pathways, we hypothesized that the combination of PI3K and MEK inhibitors might constitute an efficient treatment strategy for MBC PDX. The PI3K inhibitor BYL-719 (alpelisib) and the MEK inhibitor selumetinib were tested in monotherapy and in combination in the HBCx-60 PDX (FGFR4 amplification, PIK3CA mutation, and $A K T 1$ amplification), HBCx-165 PDX (FGFR4 amplification, PIK3CA mutation) and $\mathrm{HBCx}-178(B R A F$ mutation and PIK3CA mutation) models. Two of these models are chemoresistant to anthracycline ( $\mathrm{HBCx}-165$ and $\mathrm{HBCx}-178)$.

In the HBCx-60 model, treatment with BYL-719 and selumetinib in monotherapy did not significantly decrease tumor growth (Fig. 4a). By contrast, the combination inhibited tumor growth, with a TGI of $94 \%$ relative to the control ( $p=0.0018$, Mann-Whitney test). In this chemosensitive model, the combination of targeted therapies induced a response similar to that obtained with chemotherapy. In the HBCx-165 model (Fig. 4b), treatment with BYL-719 and selumetinib in monotherapy reduced tumor growth, with a TGI of 74\% $(p=0.028)$ and $66 \%(p=0.16)$, respectively, but xenografts showed no tumor growth arrest or regression. Conversely, in the group of xenografts treated with the 
Lousy et al. Journal of Hematology \& Oncology $\quad$ (2020) 13:13

Page 6 of 10

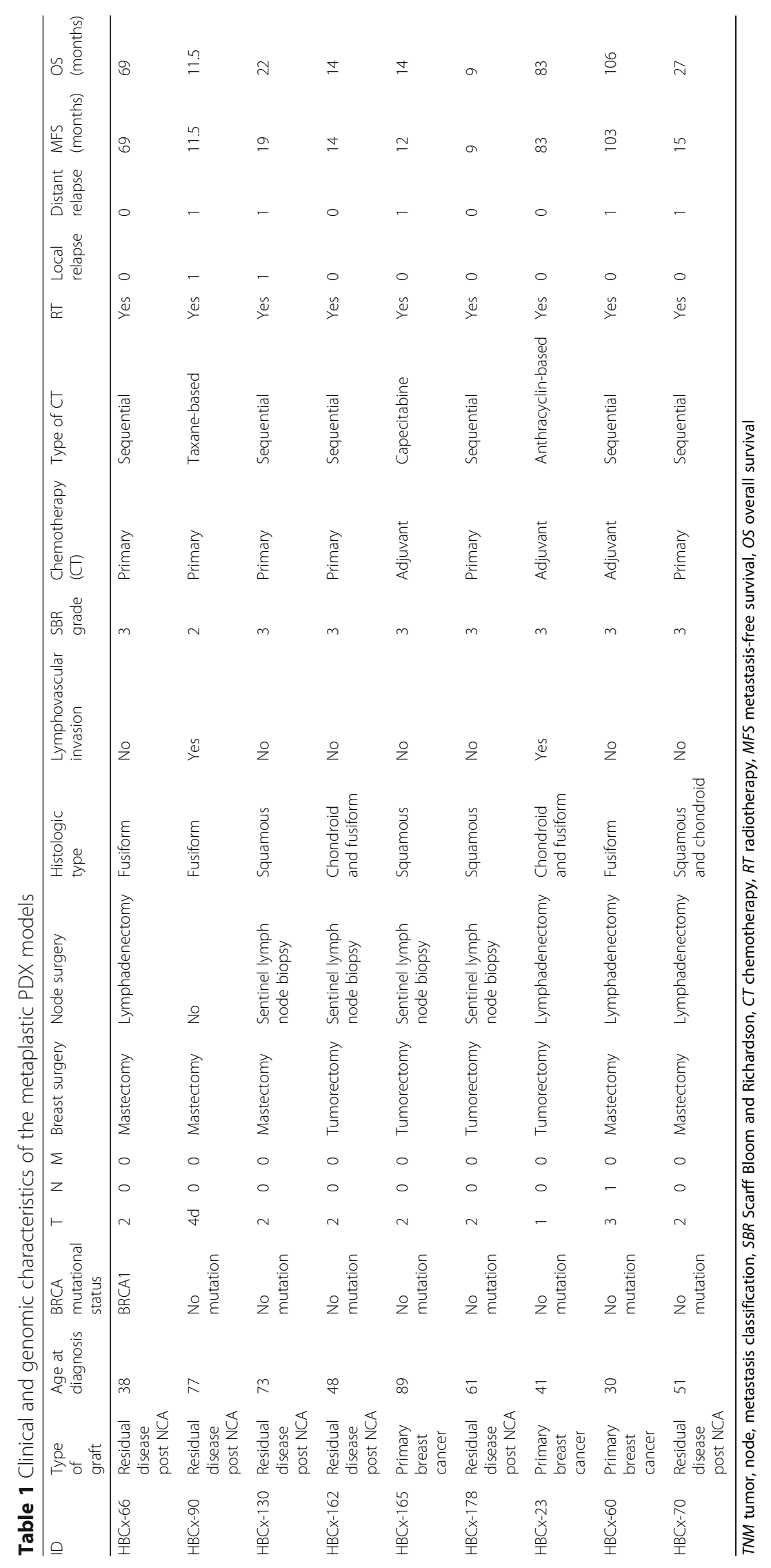




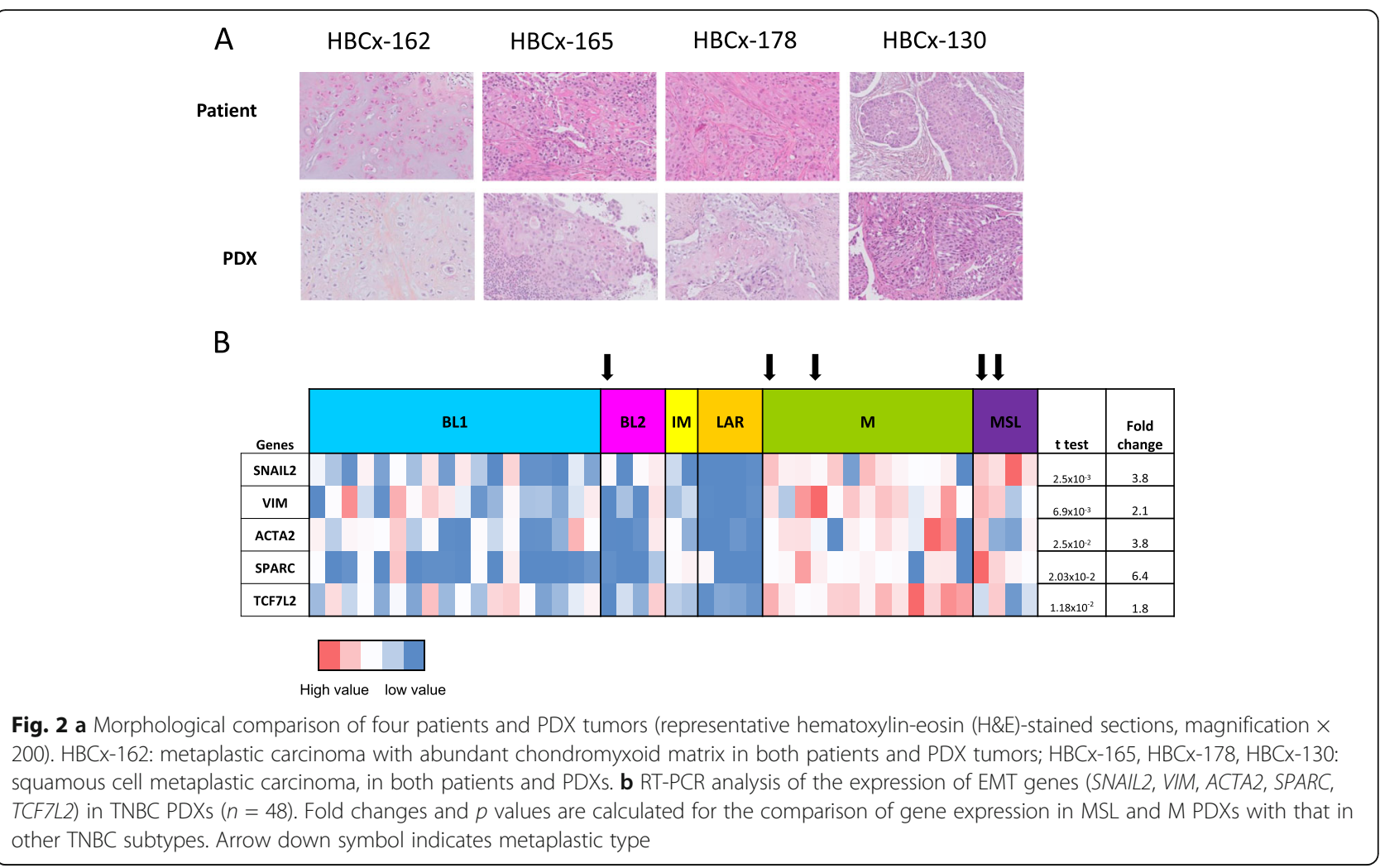

A

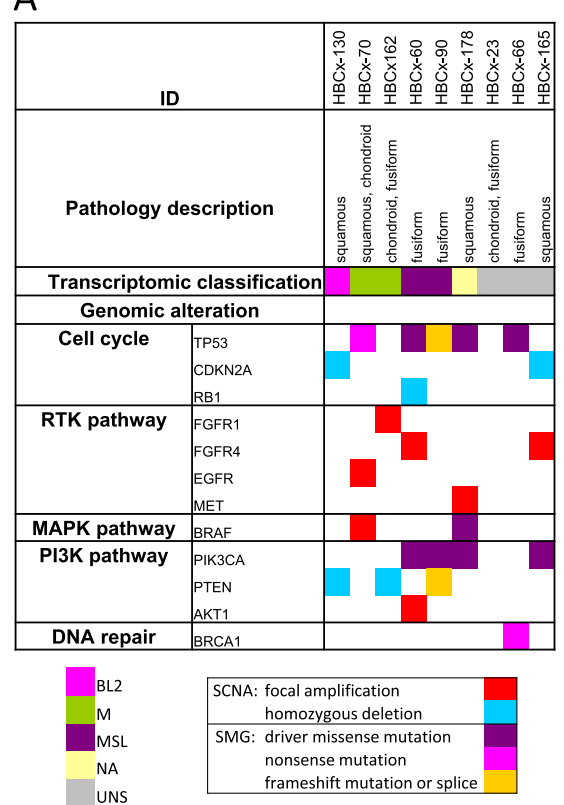

B

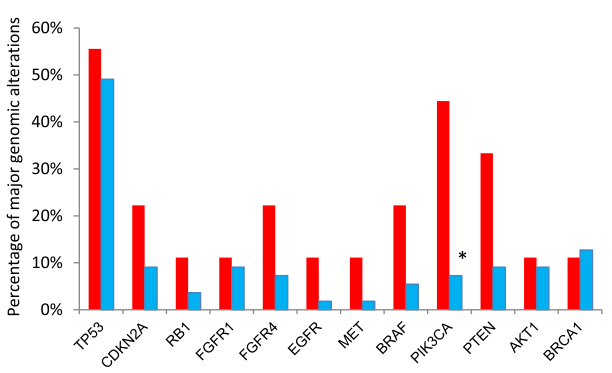

Metaplastic TNBC PDX (n=9)

Other subtypes TNBC PDX $(n=55)$

Fig. 3 Genomic alterations in metaplastic PDXs and comparison with other TNBC subtypes. a Major genomic alterations (mutations and copy number alterations) in nine metaplastic PDXs with histological characteristics and transcriptomic classification. $\mathbf{b}$ Percentage of major genomic alterations in metaplastic TNBC PDXs versus the other histologic subtypes; ${ }^{*} p=0.01$, Fisher's test 


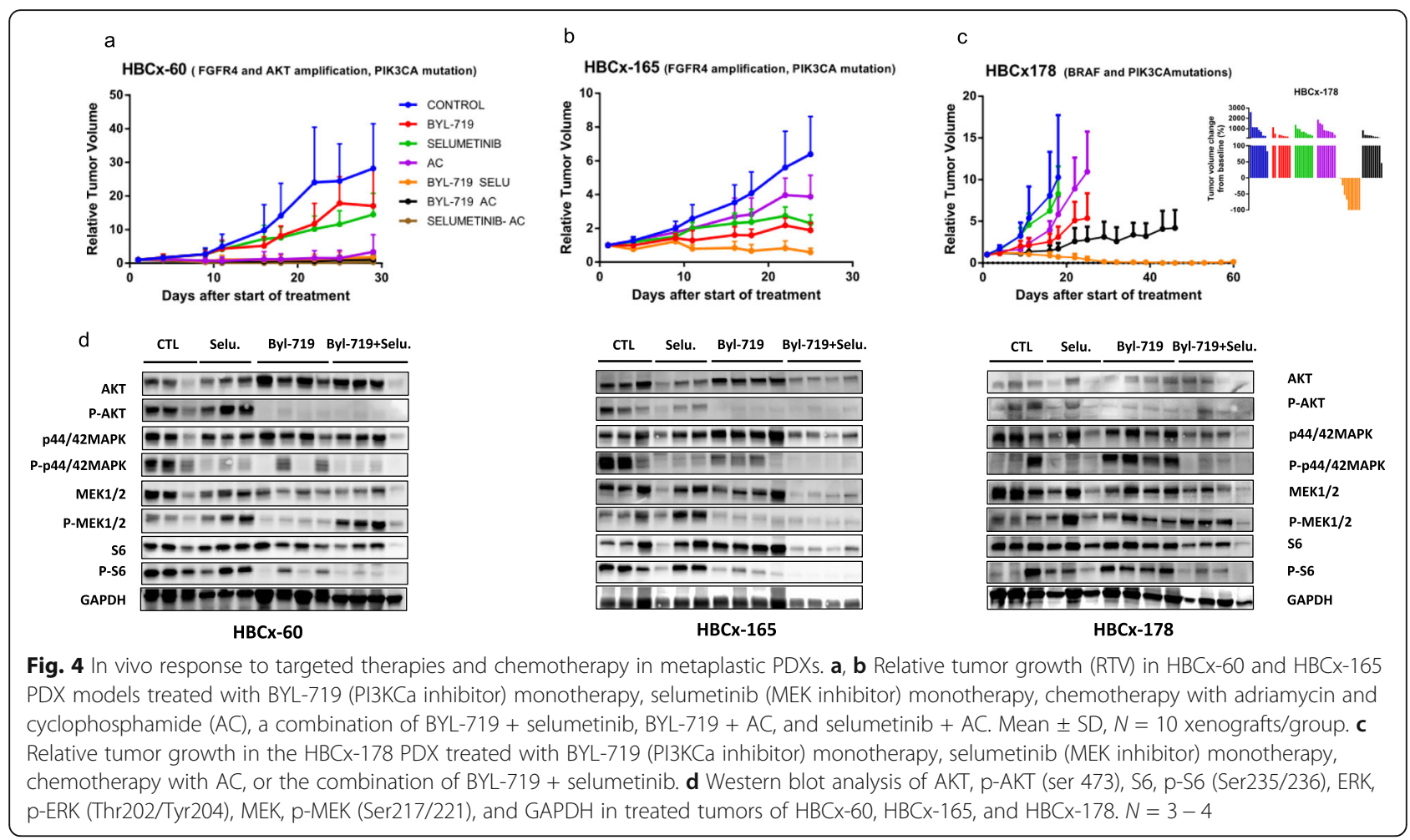

combination of BYL-719 and selumetinib (TGI of 91\%), six mice had stable disease, and two xenografts displayed tumor regression (data not shown). The combination was superior to BYL-719 alone $(p=0.0012)$. Finally, in the $\mathrm{HBCx}-178$ PDX model, treatment with BYL-719 resulted in a TGI of $70 \%$ with no tumor regression, whereas the combination of BYL-719 and selumetinib resulted in a complete response in six of ten mice (Fig. 4c).

Monotherapies induced no significant change, but the combination was highly effective, with significant tumor regression observed in all models and a complete response frequency of $60 \%$ for $\mathrm{HBCx}-178$.

To assess inhibition of PIK3 and MAPK signaling pathways, we analyzed the phosphorylation status of AKT, S6, ERK, and MEK in treated tumors harvested at the end of treatment by western blot (Fig. 4d). P-AKT was strongly inhibited in xenografts treated by BYL-719 alone or associated with selumetinib in $\mathrm{HBCx}-60$ and $\mathrm{HBCx}-165$ PDX and, to a lesser extent, in HBCx-178. PS6 was strongly inhibited in xenografts of the combination arm. In the 3 PDX models, P-ERK was inhibited in selumetinib-treated xenografts and strongly inhibited in xenografts treated with the combination BYL-719 + selumetinib. In the two models ( $\mathrm{HBCx}-60$ and $\mathrm{HBCx}$ 165), P-MEK was inhibited in BYL-719 treated tumors. Overall, these results show strong inhibition of both PIK3 and MAPK signaling pathways in tumors treated by BYL-719 associated with selumetinib.

\section{Discussion}

Metaplastic breast carcinoma constitutes a group of histologically and molecularly diverse tumors. MBC is rare, but patients with metastatic $\mathrm{MBC}$ have been shown to have lower rates of response and poorer survival [35] than patients with other subtypes and to display early relapse. Optimal treatment strategies for metastatic MBC based on genomic analysis are urgently required.

Our results confirm that (i) metaplastic breast carcinomas are mostly of the mesenchymal-like and mesenchymal stem-like TNBC subtype, according to Lehmann's classification [3], and (ii) these subtypes overexpress EMT genes. Like TNBC-NST, MBCs frequently harbor somatic TP53 mutations [13]. By contrast, MBCs more frequently display mutations affecting the PI3K-AKT-mTOR pathway $[11,12,36]$. We found that $66 \%$ of our metaplastic PDXs displayed mutations of the PI3K-AKT-mTOR pathway; a higher proportion presented PIK3CA mutations and genomic alterations affecting both the PI3K-AKTmTOR and RTK-MAPK pathways which occurred in $44 \%$ of our MBCs. This association was reported in a previous study by Krings et al. [15]. Finally, our findings confirm the activation, at the protein level, of the PI3K-AKTmTOR and, to a lesser extent, the RTK-MAPK pathway in our metaplastic PDXs.

In three metaplastic PDX models with genomic alterations to both the PI3K-AKT-mTOR and RTK-MAPK pathways, we tested a PI3K inhibitor, a MEK inhibitor, 
their combination, and their association with an anthracycline (usual chemotherapy in breast cancer). BYL-719 is an effective PI3K inhibitor because, unlike previous generations of PI3K inhibitors, its specificity for the p110 unit may be more clinically relevant and less toxic than pan-PI3K inhibition. The American Food and Drug Administration recently approved this treatment in combination with fulvestrant in metastatic settings for hormone receptor-positive, PIK3CA-mutated breast cancer. Indeed, association with BYL-719 increased median progression-free survival from 5.7 to 11 months. PI3K inhibitors are of potential clinical interest for the treatment of metaplastic $\mathrm{BC}$, as suggested by a case report from the BELLE 4 clinical trial in which a durable response obtained in a patient with metaplastic BC following treatment with a combination of buparlisib (PI3K inhibitor) and paclitaxel [37]. Nevertheless, the results for the total population were disappointing.

In two models (with PIK3Ca mutation and FGFR4 amplification), monotherapies decreased tumor growth without inducing regression. This insufficient efficacy can be explained by (i) the co-occurrence of two activating pathways. Indeed, the co-occurrence of targetable alterations has already been described in TCGA [38] and was found in $44 \%$ of our MBC PDXs, (ii) the presence of AKT1 mutation in one model, or (iii) crosstalk and compensatory mechanisms between the PI3K-AKT-mTOR and RTK-MAPK pathways [39]. By contrast, the combination of PI3K and MEK inhibitors induced some complete and durable responses in all three PDX models that were associated to strong inhibition of both PI3K and MAPK signaling pathways. In particular, in the HBCx-178 model (BRAF and PIK3CA mutation), $44 \%$ of mice presented a durable complete response. Comparison with chemotherapy highlighted (i) the superiority of the combination of two targeted therapies in chemoresistant models (HBCx165 and HBCx-178) and (ii) the similar efficacy of these two treatments in a chemosensitive model (HBCx-60).

\section{Conclusions}

Our analysis highlights the co-occurrence of actionable alterations and opportunities for combination treatment in metaplastic breast cancer. In clinical practice, the combination of specific PI3K and MEK inhibitor treatments seems to be feasible, with a manageable safety and toxicity profile (NCT01449058). A second phase I trial (NCT01449058) is currently underway, to test the safety and tolerability of BYL-719 and trametinib (MEK inhibitor). Our results provide a rationale for the genomic selection of MBCs for investigations of the use of a PI3K inhibitor in combination with a MEK inhibitor.

This work was funded by Institut Carnot and SIRIC2 (INCa-DGOS-Inserm_12554) grants.

\section{Supplementary information}

Supplementary information accompanies this paper at https://doi.org/10. 1186/s13045-020-0846-y.

Additional file 1: Table S1. Characteristics of TNBC patients.

Additional file 2: Figure S1. Genomic and proteic analysis of PI3K-AKTmTOR and RTK-MAPK pathways in PDX models.

\begin{abstract}
Abbreviations
ER: Estrogen receptor; MBC: Metaplastic breast cancer; NA: Not available; NST: No special type; PDX: Patient-derived xenografts; PR: Progesterone receptor; RPPA: Reverse-phase protein arrays; RTV: Relative tumor volume; TGI: Tumor growth inhibition; TNBC: Triple-negative breast cancer;

TSG: Tumor suppressor genes
\end{abstract}

\section{Acknowledgements}

Not applicable

\section{Authors' contributions}

FC analyzed and interpreted the PDX data and wrote the manuscript. ML performed the histological examination of patient and PDX. IB and EM supervised the study and contributed to manuscript writing. All authors read and approved the final manuscript and agreed both to be personally accountable for the author's own contributions and to ensure that questions related to the accuracy or integrity of any part of the work

\section{Authors' information}

No

\section{Funding}

This work was supported by grants from the Institut Curie, the Canceropole (PhD allocation), the Ligue contre le cancer (4FI13229SREX), and SIRIC2 (INCaDGOS-Inserm_12554).

\section{Availability of data and materials}

The datasets used and/or analyzed during the current study are available from the corresponding author on reasonable request.

\section{Ethics approval and consent to participate}

The experimental protocol and animal housing were in accordance with institutional guidelines and with the recommendations of the French Ethics Committee (Agreement B75-05-18, France).

\section{Consent for publication}

All patients admitted to our institution before 2007 were informed that their tumor samples might be used for scientific purposes and were given the opportunity to refuse such use. Since 2007, patients admitted to our institution also give express consent for the use of their samples for research purposes, by signing an informed consent form.

\section{Competing interests}

The authors declare that they have no competing interests

\section{Author details}

${ }^{1}$ Unit of Pharmacogenomics, Department of Genetics, Institut Curie, Paris, France. ${ }^{2}$ Laboratory of Preclinical Investigation, Department of Translational Research, Institut Curie Research Center, Paris, France. ${ }^{3}$ Department of Medical Oncology, Institut Curie, Paris, France. ${ }^{4}$ Department of Biopathology, Institut Curie, Paris, France. ${ }^{5}$ Translational Research Department, RPPA Platform, Institut Curie Research Center, Paris, France. ${ }^{6}$ BioPôle Alfort, National Veterinary School of Alfort, Maison Alfort, France. ${ }^{7}$ INRA, APEX-PAnTher, Oniris, Nantes, France. ${ }^{8}$ Inserm U1016, University Paris Descartes, Paris, France.

Received: 23 September 2019 Accepted: 27 January 2020

Published online: 22 February 2020

\section{References}

1. McCart Reed AE, Kalaw E, Nones K, Bettington M, Lim M, Bennett J, Johnstone K, Kutasovic JR, Saunus JM, Kazakoff S, et al. Phenotypic and 
molecular dissection of metaplastic breast cancer and the prognostic implications. J Pathol. 2019;247(2):214-27.

2. Weigelt B, Eberle C, Cowell CF, Ng CK, Reis-Filho JS. Metaplastic breast carcinoma: more than a special type. Nat Rev Cancer. 2014;14(3):147-8.

3. Weigelt B, Kreike B, Reis-Filho JS. Metaplastic breast carcinomas are basallike breast cancers: a genomic profiling analysis. Breast Cancer Res Treat. 2009;117(2):273-80.

4. Pezzi CM, Patel-Parekh L, Cole K, Franko J, Klimberg VS, Bland K. Characteristics and treatment of metaplastic breast cancer: analysis of 892 cases from the National Cancer Data Base. Ann Surg Oncol. 2007; 14(1):166-73.

5. Okada N, Hasebe T, Iwasaki M, et al. Metaplastic carcinoma of the breast. Hum Pathol. 2010;41(10):960-70.

6. Chen IC, Lin CH, Huang CS, Lien HC, Hsu C, Kuo WH, Lu YS, Cheng AL. Lack of efficacy to systemic chemotherapy for treatment of metaplastic carcinoma of the breast in the modern era. Breast Cancer Res Treat. 2011; 130(1):345-51.

7. Rayson D, Adjei AA, Suman VJ, Wold LE, Ingle JN. Metaplastic breast cancer: prognosis and response to systemic therapy. Ann Oncol. 1999;10(4):413-9.

8. Cardoso F, Bedard PL, Winer EP, Pagani O, Senkus-Konefka E, Fallowfield LJ, Kyriakides S, Costa A, Cufer T, Albain KS. ESO-MBC Task Force. International guidelines for management of metastatic breast cancer: combination vs. sequential single-agent chemotherapy. J Natl Cancer Inst. 2009;101(17): 1174-81.

9. Ong $\mathrm{CT}$, Campbell BM, Thomas SM, Greenup RA, Plichta JK, Rosenberger $\mathrm{LH}$, Force J, Hall A, Hyslop T, Hwang ES, et al. Metaplastic breast cancer treatment and outcomes in 2500 patients: a retrospective analysis of a national oncology database. Ann Surg Oncol. 2018;25(8):2249-60.

10. Weigelt B, Ng CK, Shen R, Popova T, Schizas M, Natrajan R, Mariani O, Stern MH, Norton L, Vincent-Salomon A, et al. Metastatic breast carcinomas display genomic and transcriptomic heterogeneity. Mod Pathol. 2015;28(4):340-51.

11. Hennessy BT, Gonzalez-Angulo AM, Stemke-Hale K, Gilcrease MZ, Krishnamurthy S, Lee JS, Fridlyand J, Sahin A, Agarwal R, Joy C, et al, Characterization of a naturally occurring breast cancer subset enriched in epithelial-to-mesenchymal transition and stem cell characteristics. Cancer Res. 2009;69(10):4116-24.

12. Piscuoglio S, Ng CKY, Geyer FC, Burke KA, Cowell CF, Martelotto LG, Natrajan R, Popova T, Maher CA, Lim RS, et al. Genomic and transcriptomic heterogeneity in metaplastic carcinomas of the breast. NPJ Breast Cancer. 2017;3:48.

13. Ng CKY, Piscuoglio S, Geyer FC, Burke KA, Pareja F, Eberle CA, Lim RS, Natrajan R, Riaz N, Mariani O, et al. The landscape of somatic genetic alterations in metaplastic breast carcinomas. Clin Cancer Res. 2017;23(17): 3859-70.

14. Ross JS, Badve S, Wang K, Sheehan CE, Boguniewicz AB, Otto GA, Yelensky R, Lipson D, Ali S, Morosini D, et al. Genomic profiling of advanced-stage, metaplastic breast carcinoma by next-generation sequencing reveals frequent, targetable genomic abnormalities and potential new treatment options. Arch Pathol Lab Med. 2015;139(5):642-9.

15. Krings $\mathrm{G}$, Chen YY. Genomic profiling of metaplastic breast carcinomas reveals genetic heterogeneity and relationship to ductal carcinoma. Mod Pathol. 2018;31(11):1661-74.

16. Marangoni E, Laurent C, Coussy F, El-Botty R, Château-Joubert S, Servely JL, de Plater L, Assayag F, Dahmani A, Montaudon E, et al. Capecitabine efficacy is correlated with TYMP and RB1 expression in PDX established from triple-negative breast cancers. Clin Cancer Res. 2018;24(11):2605-15.

17. Coussy F, Lallemand F, Vacher S, Schnitzler A, Chemlali W, Caly M, Nicolas A, Richon S, Meseure D, El Botty R, et al. Clinical value of Rspondins in triple-negative and metaplastic breast cancers. $\mathrm{Br} J$ Cancer. 2017;116(12):1595-603.

18. Marangoni E, Vincent-Salomon A, Auger N, Degeorges A, Assayag F, de Cremoux P, de Plater L, Guyader C, De Pinieux G, Judde JG, et al. A new model of patient tumor-derived breast cancer xenografts for preclinical assays. Clin Cancer Res. 2007;13(13):3989-98.

19. Carvalho $B S$, Louis TA, Irizarry RA. Quantifying uncertainty in genotype calls. Bioinformatics. 2010;26(2):242-9.

20. Samuels AL, Peeva VK, Papa RA, Firth MJ, Francis RW, Beesley AH, Lock RB, Kees UR. Validation of a mouse xenograft model system for gene expression analysis of human acute lymphoblastic leukaemia. BMC Genomics. 2010;11:256.
21. Chen X, Li J, Gray WH, Lehmann BD, Bauer JA, Shyr Y, Pietenpol JA. TNBCtype: a subtyping tool for triple-negative breast cancer. Cancer Inform. 2012;11:147-56

22. Coussy F, de Koning L, Lavigne M, Bernard V, Ouine B, Boulai A, El Botty R, Dahmani A, Montaudon E, Assayag F, et al. A large collection of integrated genomically characterized patient-derived xenografts highlighting the heterogeneity of triple-negative breast cancer. Int J Cancer. 2019;145(7): 1902-12.

23. Laurent C, Gentien D, Piperno-Neumann S, Némati F, Nicolas A, Tesson B, Desjardins L, Mariani P, Rapinat A, Sastre-Garau X, et al. Patient-derived xenografts recapitulate molecular features of human uveal melanomas. Mol Oncol. 2013;7(3):625-36.

24. Crepin R, Gentien D, Duche A, Rapinat A, Reyes C, Némati F, Massonnet G, Decaudin D, Djender S, Moutel S, et al. Nanobodies against surface biomarkers enable the analysis of tumor genetic heterogeneity in uveal melanoma patient-derived xenografts. Pigment Cell Melanoma Res. 2017; 30(3):317-27.

25. Gao J, Aksoy BA, Dogrusoz U, Dresdner G, Gross B, Sumer SO, Sun Y, Jacobsen A, Sinha R, Larsson E, et al. Integrative analysis of complex cancer genomics and clinical profiles using the cBioPortal. Sci Signal. 2013;6:269.

26. Cerami E, Gao J, Dogrusoz U, Gross BE, Sumer SO, Aksoy BA, Jacobsen A Byrne CJ, Heuer ML, Larsson E, et al. The cBio cancer genomics portal: an open platform for exploring multidimensional cancer genomics data. Cancer Discov. 2012:2:401-4.

27. Olshen $A B$, Venkatraman ES, Lucito R, Wigler M. Circular binary segmentation for the analysis of array-based DNA copy number data. Biostatistics. 2004;5(4):557-72

28. Mermel CH, Schumacher SE, Hill B, Meyerson ML, Beroukhim R, Getz G. GISTIC2.0 facilitates sensitive and confident localization of the targets of focal somatic copy-number alteration in human cancers. Genome Biol. 2011;12(4):R41.

29. Lingjaerde OC, Baumbusch LO, Liestol K, Glad IK, Borresen-Dale AL. CGHExplorer: a program for analysis of array-CGH data. Bioinformatics. 2005; 21(6):821-2

30. Popova T, Manie E, Rieunier G, Caux-Moncoutier V, Tirapo C, Dubois T, Delattre O, Sigal-Zafrani B, Bollet M, Longy M, et al. Ploidy and large-scale genomic instability consistently identify basal-like breast carcinomas with BRCA1/2 inactivation. Cancer Res. 2012;72:5454-62.

31. Bieche I, Parfait B, Le Doussal V, Olivi M, Rio MC, Lidereau R, Vidaud M. Identification of CGA as a novel estrogen receptor-responsive gene in breast cancer: an outstanding candidate marker to predict the response to endocrine therapy. Cancer Res. 2001;61(4):1652-8.

32. Bieche I, Onody P, Laurendeau I, Olivi M, Vidaud D, Lidereau R, Vidaud M. Real-time reverse transcription-PCR assay for future management of ERBB2based clinical applications. Clin Chem. 1999:45:1148-56.

33. Rondeau S, Vacher S, De Koning L, Briaux A, Schnitzler A, Chemlali W, Callens C, Lidereau R, Bièche I. ATM has a major role in the double-strand break repair pathway dysregulation in sporadic breast carcinomas and is an independent prognostic marker at both mRNA and protein levels. Br J Cancer. 2015;112:1059-66.

34. Zhang Y, Toy KA, Kleer CG. Metaplastic breast carcinomas are enriched in markers of tumor-initiating cells and epithelial to mesenchymal transition. Mod Pathol. 2012;25(2):178-84

35. Liao HY, Zhang WW, Sun JY, Li FY, He ZY, Wu SG. The clinicopathological features and survival outcomes of different histological subtypes in triplenegative breast cancer. J Cancer. 2018;9(2):296-303.

36. Zhai J, Giannini G, Ewalt MD, Zhang EY, Invernizzi M, Niland J, Lai LL, et al. Molecular characterization of metaplastic breast carcinoma via nextgeneration sequencing. Hum Pathol. 2019;86:85-92.

37. Yang MH, Chen IC, Lu YS. PI3K inhibitor provides durable response in metastatic metaplastic carcinoma of the breast: a hidden gem in the BELLE4 study. J Formos Med Assoc. 2019;1 18:1333-8.

38. Sanchez-Vega F, Mina M, Armenia J, Chatila WK, Luna A, La KC, Dimitriadoy S, Liu DL, Kantheti HS, Saghafinia S, et al. Oncogenic signaling pathways in the cancer genome atlas. Cell. 2018;173:321-37.

39. Mendoza MC, Er EE, Blenis J. The Ras-ERK and PI3K-mTOR pathways: crosstalk and compensation. Trends Biochem Sci. 2011;36:320-8.

\section{Publisher's Note}

Springer Nature remains neutral with regard to jurisdictional claims in published maps and institutional affiliations. 